https://doi.org/10.18778/2196-8403.2020.06

\title{
ANNA-KATHARINA GISBERTZ
}

\section{Familienverflechtungen. Im Gespräch mit NATASCHA WODIN}

NATASCHA WODINS Familiengeschichte beleuchtet das überwältigende Ausmaß der Zwangsarbeit in Deutschland während der Zeit des Nationalsozialismus. Dieser Teil der Geschichte wurde im kulturellen Gedächtnis der Deutschen bislang vernachlässigt. Im vorliegenden Interview erörtert die Autorin die Hintergründe über die Recherche und Arbeit an ihren letzten beiden Generationserzählungen Sie kam aus Mariupol (2017) und Irgendwo in diesem Dunkel (2018). WODIN rekapituliert das schwierige Leben mit ihrer Familiengeschichte und gibt Einblick in ihre Schreibstrategien. Eine Einführung in WODINS Werk geht dem Gespräch voran.

Schlüsselwörter: NATASCHA WODIN, literarische Biographie, Trauma, russische Zwangsarbeit, europäische Identität, kulturelles Gedächtnis

\section{Family links. NATASCHA WODIN in conversation}

NATASCHA WODIN's family story illuminates the overwhelming extent of forced labour in Germany during the years of National Socialism. This aspect of history has been neglected so far in the German cultural memory. In this present interview the author presents more information about her research and her work on her last two generational stories She Came from Mariupol (2017) and Anywhere in this Darkness (2018). WoDIN recalls the problematic life with her family history and introduces us to her writing strategies. The interview is preceded by an introduction to her work.

Keywords: NATASCHA WODIN, Literary Biography, Trauma, Forced Labor of Russions, European Identity, Cultural Memory

\section{Powiązania rodzinne. Rozmowa z NATASCHĄ Wodin}

Historia rodziny NATASCHY WODIN rzuca światło na skalę zjawiska pracy przymusowej w nazistowskich Niemczech. Ten aspekt historii Niemiec jest jak dotąd nieprzepracowany przez pamięć kulturową. Autorka ujawnia w publikowanym wywiadzie szczegóły, 
dotyczące poszukiwań dokumentów i pracy nad jej ostatnimi opowiadaniami Sie kam aus Mariupol (2017) / Przybyla z Mariupola oraz Irgendwo in diesem Dunkel (2018) / Gdzieś w tej ciemności. WoDIN wspomina, jak trudno było jej żyć z własną historią rodzinną i opowiada o swym warsztacie pisarskim. Zapis rozmowy poprzedzony jest wprowadzeniem do twórczości NATASCHY WODIN.

Stowa klucze: NATASCHA WODIN, biografia, trauma, praca przymusowa, Rosja, tożsamość europejska, pamięć kulturowa

\section{Zur Einführung}

NATSCHA WODIN wurde auf der Schattenseite des Lebens geboren und hatte Gewalt, Unterdrückung, Ausgrenzung viel zu früh erlebt. Als Kind wünschte sich die Autorin in das Leben hinein, das andere wie selbstverständlich führten. Sie blieb jedoch zunächst eine Außenseiterin, die sich den Umständen nicht fügte, sondern zu schreiben begann: Zuerst waren es Phantasie- und Fluchtgeschichten, schließlich ein eigenes Werk. Das Schreiben dient als Rückzugsort und als Verbindung mit der Welt, als Abenteuer neuer Welten und manchmal auch als ein Überlebensmittel. Besonders eindrücklich prägt WODINS sensible Auseinandersetzung mit dem eigenen Leben ihre Literatur. Indem die Autorin von ihrem Leben erzählt, verwebt sie ganz Persönliches - präzise beobachtend und sehr vertraut mit den Abgründen der menschlichen Seele - mit Angst, Scham, Depression - mit den Bildern, die wir uns machen und der Andersartigkeit der Realität.

Ihre Biographie erschöpft sich von außen gesehen in nur wenigen Daten: WODIN wird 1945 in Fürth (Bayern) geboren, ist Kind sowjetischer Zwangsarbeiter und wächst erst in einer alten Scheune, später in einem Lager für Displaced Persons und schließlich am Stadtrand von Fürth auf. ${ }^{1}$ Als sie zehn Jahre alt ist, bringt sich ihre Mutter um. Nach Stationen in einer Pflegefamilie, einem Waisenhaus und einem Mädchenheim kehrt sie mit ihrer Schwester zurück zum Vater, der in einem russischen Kosakenchor singt. Er bewältigt das Familienleben nicht. Mit siebzehn Jahren flieht sie vor seiner Gewalt in die Obdachlosigkeit, anschließend in eine zu frühe Ehe, bis sie Studentin wird und sich ein eigenes Leben aufbaut. Sie studiert Russisch, arbeitet als Übersetzerin und schließt sich literarischen Kreisen in Moskau an. 1983 folgt ihr Romandebüt Die gläserne Stadt. Anschließend veröffentlicht sie die ersten Gedichte, Erzählungen und Romane,

1 Vgl. den den wikipedia-Eintrag über NATASCHA WODIN oder das Autorenprofil im Rowohlt Verlag. https://de.wikipedia.org/wiki/Natascha_Wodin; https://www.rowohlt.de /autor/natascha-wodin.html. 
wozu Erfindung einer Liebe (1993), Die Ehe (1997), Das Singen der Fische (2001) und Nachtgeschwister (2009) gehören. Inzwischen lebt sie in Berlin und Mecklenburg und kann auf zahlreiche Ehrungen und Preise zurückblicken: den Hermann-Hesse-Preis, den Brüder-Grimm-Preis, den Adelbert-von-ChamissoPreis, den Alfred-Döblin-Preis und den August-Graf-von-Platen-Preis, um nur einige zu nennen. Der größere Ruhm erreicht sie im Alter durch ihre Generationsgeschichte Sie kam aus Mariupol (2017), die mit dem Preis der Leipziger Buchmesse ausgezeichnet wurde. Für Irgendwo in diesem Dunkel (2018) wurde ihr zuletzt der Hilde Domin-Preis verliehen.

Was die äußeren Daten nicht mitteilen, steht in WoDINS Büchern, die einen Kosmos dessen eröffnen, was lieber nicht hätte sein dürfen. Es ist ein Stück deutscher Geschichte aus der Sicht einer Außenseiterin. Als Deutsche und Russin erlebt die Ich-Erzählerin eine abschreckende Schulzeit, in der sie das Feindbild der Russen verkörpert und von ihren Mitschülern verfolgt und verprügelt wird. Das Elternhaus ist durch die Erfahrung der Zwangsarbeit entwurzelt und von Gewalt geprägt. Im katholischen Mädcheninternat ist sie als RussischOrthodoxe eine „negative Besonderheit“ (WODIN 2018) und als Jugendliche eine Nicht-Deutsche, die gern wie die Deutschen sein will. Das Versagen der Institutionen und der Menschen zeigt sich nicht als individuelles Problem, sondern reicht zurück in die düstere Geschichte der von Krieg und Revolution gezeichneten Generationen des 20. Jahrhunderts. Die Forschung zu WODINS Werk steckt entsprechend in ihren Anfängen. ${ }^{2}$

Die beiden letzten Bücher sind dem Rückblick auf die Kindheit und dem Leben der Eltern gewidmet. Sie kam aus Mariupol (2017) schildert die Familiengeschichte von NATASCHA WODINS Mutter Jewgenia Jakowlewna Iwaschtschenko, über die die Tochter intensiv recherchiert hat und eine zentrale Leerstelle im kulturellen Gedächtnis in Deutschland füllt - stammte die Mutter doch aus einer adligen Familie, die aufgrund der Revolution von Beginn an unter Verfolgung, Hunger und Ausstoß litt (vgl. WODIN 2017). Jewgenia wurde 1920 geboren und 1943 mit ihrem Mann aus der Ukraine zur Zwangsarbeit nach Deutschland verschleppt. Zehn Jahre nach dem Zweiten Weltkrieg, die sie aus Angst vor der Verfolgung durch die Russen weiterhin in Deutschland verbrachte, beging sie Selbstmord. Um den Zwangsarbeiterinnen und Zwangsarbeitern, die für die Industrie und auf dem Land millionenfach missbraucht wur-

2 Erste Forschungsarbeiten zu Einmal lebt ich und Die gläserne Stadt bieten neben Aufsätzen in ihren Buchkapiteln z.B. SUREN (2011) und HoGE-BENTELER (2012). 
den, Gehör zu verschaffen, ist das Buch seither eine wichtige Stimme geworden. ${ }^{3}$ Indem WoDIN das Schweigen über die Zeit bricht, unter der sie selbst gelitten hat, wurde ihr Werk an der Grenze von Fiktion und Nicht-Fiktion eingestuft und von der Jury für den Leipziger Buchpreis als „unerhört zeitgenössisch" angesehen. ${ }^{4}$

Mit Irgendwo in diesem Dunkel (2018) liegt der Folgeroman vor, der sich dem Vater widmet und aus der Zeit zwischen WoDINs Geburt und der Flucht aus der väterlichen Enge und Gewalt erzählt. Die Ereignisse werden nicht chronologisch gegliedert, sondern kehren als Erinnerungen an das lange Sterben des über neunzig Jahre alten Vaters zurück, der bis zum Tod, schwer gebrechlich, in einem Altenheim lebte. Das Problem der Familie, die unter häuslicher Gewalt durch den Vater litt, wird im Zusammenhang mit den Kriegserlebnissen neu geordnet und nach Schuld und Verantwortung gefragt. Der Vater erscheint als Opfer und Täter zugleich, weil er erlebte Gewalt auch weitergegeben hat. Als Buch des Monats wurde Irgendwo in diesem Dunkel bereits vom ORFHörfunk mit der Begründung vorgeschlagen, es sei „der stille, zurückhaltende Triumph einer Autorin über die Traumata ihrer Biografie. "5 Ihre Themen bilden eine Realität ab, die aufgrund von Schrecken, Gewalt oder Irrsinn schlichtweg nicht begreifbar sein kann. Über das Thema der Familie als Generationserzählung, die Leerstellen der Geschichte und die Bedeutung des Schreibens sprechen NATASCHA WODIN und ANNA-KATHARINA GiSBERTZ. ${ }^{6}$

3 Der historischen Erforschung der Zwangsarbeit 1939-1945, ihrer Archivierung, Digitalisierung und dem Aufbau einer Online-Plattform widmet sich z.B. die Freie Universität Berlin unter https:/www.zwangsarbeit-archiv.de. Die Plattform enthält ein mehrsprachiges Interview-Archiv, Kurzfilme und eine didaktische Aufbereitung des Themas.

JURY DES LEIPZIGER BUCHPREISES (2017): Begründung der Preisträgerin 2017 - Natascha Wodin. http://www.preis-der-leipziger-buchmesse.de/de/Archiv/2017/ (29.01.2020). Zum Interview über die Zwangsarbeit vgl. z.B. SCHMIDTKUNZ (2017).

NATASCHA Wodins Irgendwo in diesem Dunkel ist Ö1 Buch des Monats Dezember. Pressemitteilung des Rowohlt-Verlags vom 26.11.2018. https://www.rowohlt.de/news /natascha-wodin-irgendwo-in-diesem-dunkel-ö1-buch-des-monats-dezember.html (29.01.2020).

Das Gespräch entstand im Januar 2020 im Rahmen einer Lesung aus der Reihe „Europa Morgen Land“ in Mannheim. Nähere Informationen unter www.europamorgenland.de. 


\section{Familienverhältnisse - Irgendwo in diesem Dunkel (2018). Ein Gespräch}

GISBERTZ: Seit wann wollten Sie über Ihren Vater schreiben? Und ab wann konnten Sie es?

WoDIN: Ich habe schon einmal in Einmal lebt ich (1989) - einem Zitat aus Hölderlins An die Parzen - über meinen Vater und meine Geschichte mit ihm geschrieben. Es war jedoch ein ganz anderes Buch, da ich noch nichts über meine Familiengeschichte wusste. Ich habe ihn immer aus der Sicht des Kindes gesehen. Und nachdem ich mein Buch über meine Mutter geschrieben habe, fragten mich viele Leute, warum mein Vater keine Rolle spielte. Ich wollte zunächst nicht noch einmal über meinen Vater schreiben, aber als auch der Verlag mich danach fragte, überlegte ich noch einmal und kam zu dem Schluss, dass ich meinem Vater tatsächlich etwas schuldig geblieben bin. Ich habe ihn nie aus dem Kinderblick entlassen und nie versucht zu verstehen, warum er so war, wie er war.

GISBERTZ: Sie entschuldigen ihn nicht für seine Gewalt Ihnen gegenüber und zeigen auch Ihren Hass gegenüber dem Vater. Umso erstaunlicher ist es, dass Sie sich im Alter dennoch um ihn kümmern. Gibt es vielleicht immer zwei Väter, den echten und den erwünschten, dessen gutes Kind man bleiben möchte? Haben Sie vielleicht auch ein wenig um den Vater getrauert, den Sie nicht hatten?

WODIN: Es liegt eigentlich nahe, dass es so gewesen sein könnte. Aber ich habe meinen Vater eigentlich nie vermisst, sondern war nur auf der Flucht vor ihm. ,Vater' war für mich keine Gestalt, die ich mir anders vorstellen konnte als so, wie er gewesen ist. Ich habe ihn dann im Alter sehr lange leiden sehen - 20 Jahre fast - sodass ich Mitleid bekam, ob ich wollte oder nicht. Das kommt automatisch. Der Hass ist deswegen aber nicht weggegangen. Das hat beides in mir koexistiert und war ungut. In dem Buch habe ich versucht, das auszugleichen und mich mit meinem Vater zu versöhnen, aber ich weiß nicht, ob mir das wirklich gelungen ist.

GISBERTZ: Während die Leserinnen und Leser in Ihrem Buch Sie kam aus Mariupol Ihre detaillierte Recherchearbeit verfolgen können, die wie eine Detektivgeschichte immer tiefer in Ihre Familienverhältnisse und das Schicksal der Ukrainerinnen und Ukrainer im 20. Jahrhundert hineinreicht, fehlt die Form der 
Recherche in Ihrem Vater-Buch. Welchen Stellenwert hatte die Recherchearbeit diesmal für Ihr Schreiben?

WoDIN: Ich wollte nicht noch ein Recherchebuch schreiben. Das Buch über meine Mutter war ein Buch über das Finden. Es war ein sehr überraschendes Finden: Es ist wie vom Himmel auf mich gefallen, denn ich war schon über 70 Jahre alt, habe jahrzehntelang nach meiner Mutter gesucht und sie nicht gefunden. Und eines Tages gab ich Ihren Namen im Internet ein und fand sie auf einmal. Wie eine Lawine kamen die Informationen auf mich zu. Das war wie ein Wunder in meinem Leben. Im Buch über meinen Vater habe ich gar nicht recherchiert. Das lag mir fern. Es ist eher der Versuch des Findens, aber Nicht-Finden-Könnens. Der Vater bleibt im Dunkel. Es sind immer diese kreisenden Suchbewegungen nach ihm, aber sie führen letztlich zu sehr wenig.

\section{GISBERTZ: Können Sie das Nicht-Finden-Können näher beschreiben?}

WodIN: Mein Vater hatte vor uns eine andere Familie. Davon hatte ich überhaupt keine Ahnung. Das war für mich eine überwältigende Überraschung, dass mein Vater in der Sowjetunion schon mal verheiratet gewesen ist. Aber ich weiß nichts über diese Familie. Der Bruder meines Vaters hat mir nichts verraten - ich kam nicht an das Geheimnis heran.

GISBERTZ: Das Verhältnis Ihres Vaters zur Mutter war sehr hierarchisch und von Gewalt geprägt. Sie thematisieren die Rolle der Frau folglich in düsterem Licht: ausgenutzt, missbraucht, vergewaltigt. Zum anderen hatten Sie selbst stets den Drang, herauszukommen. Woher kam diese Kraft zur Emanzipation?

WoDIN: Ich weiß nicht, ob es ein Drang zur Emanzipation war. Es war eher ein Drang, so zu sein wie alle. Das ist bei Kindern wohl stark ausgeprägt, und bei mir war es vielleicht besonders ausgeprägt. Jedenfalls hat es mich immer beherrscht. Ich wusste schon seit sehr früher Zeit, dass das Leben, das ich führte, irgendwo draußen war. Ich sah, dass es eine andere Welt gab, zu der wir nicht gehörten. Ich sah aber auch, dass das die richtige Welt war, die legale Welt; die Welt, in der es den Menschen gut ging, die besser war als wir. Davon war ich überzeugt als Kind. Und dahin wollte ich. Ich hatte das Gefühl, ich sei in irgendeinem Irrtum gefangen und gehörte in Wirklichkeit zu den Deutschen. Ob das sehr emanzipatorisch war, bezweifle ich. 
GISBERTZ: Das Buch erzählt auch von Ihrer Jugend, der Obdachlosigkeit und endet mit Ihrem ersten Job. Wie schafften Sie es, sich nach Ihrer Flucht vom Vater auf die Straße ein Leben aufzubauen und viele Jahre später ihren ersten Roman zu veröffentlichen?

WODIN: Die Obdachlosigkeit ist recht wundersam zu Ende gegangen. Ich fand sozusagen von der Straße eine Arbeit. Ich wurde Telefonistin, was für mich großartig war. Das Telefon war damals noch ziemlich neu. Dann hat mich ein deutscher Mann geheiratet, ob zu meinem Glück oder Unglück, weiß ich nicht. Mit dem Mann bin ich aus der Kleinstadt herausgekommen. Wir sind nach München gezogen, und dort habe ich sehr viel Neues kennengelernt. Und in den Jahren, als Willy Brandt die Ostverträge unterzeichnet hat und sich Wirtschaftsbeziehungen zu Russland anbahnten, kam ich auf die Idee Russisch zu studieren und Dolmetscherin zu werden.

\section{Poetisches und historisches Schreiben}

GISBERTZ: Sprechen wir über Ihre schriftstellerische Arbeit. Was bedeutet Ihnen das Schreiben? Erfüllt es angesichts der autobiographischen Bezüge für Sie auch eine therapeutische Funktion?

WodIN: Das Schreiben ist keine Therapie, sondern eine schwere Arbeit, die man sich antut. Man kann unter Umständen auch depressiv davon werden. Denn ich sitze manchmal vor einer verschlossenen Tür und weiß nicht, wie ich in den Text reinkomme oder wie ich ihn weiterführen soll. Eine Therapie ist das höchstens insofern, als ich versuche, den Dingen eine Form zu geben. Bevor ich anfange zu schreiben, muss etwas innerlich schon geklärt sein, sonst kann ich nicht schreiben. Ich muss dafür die Worte und die Form gefunden haben. Das ist ja die Arbeit beim Schreiben. Und was eine Form hat, wird sofort sehr viel weniger bedrohlich. Alles, was frei herumschwebt und flottiert, ist das Gegenteil. Es hilft dann auch der Psyche, mit den Dingen umzugehen.

GISBERTZ: Sie schreiben in Form der Erinnerung, die durch die Ich-Erzählerin auch sehr authentisch wirkt. Man glaubt Ihren Büchern jedes Wort. Im Auslobungstext des Leipziger Buchpreises wird Ihr ,autobiografisches Schreiben mit einem hohen Maß an Selbstreflexion und romanhaftes Schreiben auf der Grundlage von Lidias Tagebüchern" positiv hervorgehoben (JURY DES LEIPZIGER BUCHPREISES 2017). Wieviel Platz lassen Sie der Fiktion? 
Wodin: Mein Mutter-Buch ist ein Buch, bei dem ich mich bemüht habe, so viel Realität und Wahrheit wiederzugeben wie möglich. Darum bemühe ich mich in meinen anderen Büchern nicht. Ich überlasse mich der Erinnerung, meinen Gedanken. Und die führen mich manchmal zu Dingen, die so hätten sein können, aber so nie waren. Manchmal produzieren Gefühle auch Bilder und Ereignisse, die es so nicht gegeben hat. Aber alles, was ich schreibe, wurzelt in Erlebtem.

GISBERTZ: Die Einbildungskraft, heißt es an einer Stelle, helfe der Ich-Erzählerin, nicht wahrzunehmen, wer sie ist und wie sie wirklich aussieht. Sie könne mühelos in andere Personen hineinschlüpfen. Sie brauche sich nur kurz in Fotos zu versenken, und schon sei sie „Nadja Tiller, Lili Palmer oder Sophia Loren“ (WODIN 2018:202). Ist das autobiographisch zu verstehen?

WODIN: Ja, ich glaube, ich kann das ganz gut. Jedenfalls konnte ich es als Kind ganz gut. Wenn ich im Kino war, war ich eine Woche lang die Heldin dieses Films. Das war das andere, die Welt, in die ich wollte. Und dann habe ich die Heldin dieses Films gespielt und auch das Gefühl gehabt, ich sei Lili Palmer.

GISBERTZ: Wofür Sie ja vor allem eine Form gefunden haben, ist die Geschichte der Zwangsarbeit. Das Interesse daran ist durch Ihre Bücher gewachsen. Sie sind zu einer Stimme für die Millionen Toten und Gequälten aus Russland und der Ukraine geworden. Hat sich etwas in der Bereitschaft des Publikums hier geändert, sich solcher Geschichten anzunehmen? Oder wäre es auch schon früher möglich gewesen, sie zu erzählen?

WodIN: Ich weiß gar nicht, ob es heute ein Thema ist. Das Buch Sie kam aus Mariupol hatte eine Resonanz, mit der ich in der Tat nie gerechnet habe. Es wurde darüber viel geschrieben. Und es kommen viele Menschen zu den Lesungen, was mich freut. Aber ich vermisse nach wie vor den Umgang damit in den Medien. Es ist zwar darüber geforscht worden, und vieles ist bekannt. Aber sehr viele wissen darüber heute noch immer wenig Bescheid. Das Ausmaß ist nicht bekannt. Wenn ich meine Freunde frage, wie viele Lager es im Nationalsozialismus gegeben hat, denken sie an 20 oder auch 200. In Wirklichkeit waren es 42000. Und 35000 davon waren Zwangsarbeiterlager. Jeder hatte also ein Lager vor der Tür. Es war nicht zu übersehen, dass diese Millionen Menschen im Land waren und schufteten. Aber es wurde ja auch nichts weitergegeben. Die Eltern 
haben nach dem Krieg geschwiegen. Und die Zwangsarbeiter gehörten wahrscheinlich so selbstverständlich zum Stadtbild, dass man sie kaum als irgendein besonderes Phänomen wahrgenommen hat.

GISBERTZ: Sehen Sie sich in der Auseinandersetzung mit dem Thema als Nachgeborene oder eher als direkte Zeugin des Geschehens? Was ich meine ist, dass Sie sich als Tochter von Zwangsarbeitern ja deutlich zu erkennen geben und somit auch vielleicht klarer als andere auf eine Leerstelle in der offiziellen Geschichtsschreibung hinweisen können.

WODIN: Ja, wenn man so will. Ich habe es nicht mit diesem Impetus geschrieben, ich wollte über die Zwangsarbeit schreiben, wusste aber noch nicht, wie ich es machen sollte. Dann dachte ich mir, ich müsse einfach die Geschichte meiner Mutter erfinden, um an den historischen Ereignissen entlang über die Zwangsarbeit zu erzählen. Dann habe ich meine Mutter gefunden und konnte darüber erzählen. Ihre Geschichte war auch eine Folie, um über die Zwangsarbeit zu erzählen. Ich war davon überzeugt, dass es meine Pflicht war. Ich musste sagen, dass es nicht nur den Holocaust gegeben hat, sondern auch das historische Verbrechen der Zwangsarbeit.

GISBERTZ: Warum konnten Sie Ihre Mutter nach der langen Suche im russischen Internet plötzlich finden?

WODIN: Es ist tatsächlich verwunderlich, warum eine bedeutungslose Frau, eine Zwangsarbeiterin, die sich vor sechs Jahrzehnten in Deutschland das Leben genommen hat, im russischen Internet auftaucht. Ich habe meinen Augen nicht getraut. Im Laufe meiner Recherche hat sich herausgestellt, dass meine Mutter einen berühmten Verwandten hatte. Eine Tante von ihr war mit ihm verheiratet, er war Philosoph und der erste russische Experimentalpsychologe. Und meine Mutter war eine Nichte von ihm. Hätte diese Tante den Mann nicht geheiratet, hätte ich wohl nie etwas über sie herausgefunden. Der Interneteintrag galt dem berühmten Mann und seiner gesamten Familie.

GISBERTZ: Und nun sind Sie berühmt: Wie steht es mit dem Druck Ihres schriftstellerischen Tuns, der nach diesem Erfolg entstand? 
WoDIN: Der Erfolg hat mein Leben stark verändert. Ich habe jetzt sehr viele Verpflichtungen, die ich vorher, als ich mich eher am Rand des Literaturbetriebs aufhielt, nicht hatte. Ich gehörte ganz mir selbst. Jetzt muss ich manchmal aufpassen, dass ich mich nicht verliere im Gedränge. Und ich muss mich allmählich wieder auf meine eigentliche Aufgabe besinnen, auf das Schreiben. Trotzdem bin ich sehr dankbar für den Erfolg, dafür, dass so viele Menschen das Buch über meine Mutter gelesen haben, und nicht zuletzt hat es mir so etwas wie eine Rente eingebracht und das Gespenst der Altersarmut gebannt.

GISBERTZ: Sie erreichen mit Ihren Büchern ein deutsches Publikum, das sich auch damit auseinandersetzt. Wie nimmt aber das russische und ukrainische Publikum Ihre Bücher auf?

Wodin: Sie kam aus Mariupol und Irgendwo in diesem Dunkel wurden nicht ins Russische übersetzt, das erstere aber ins Ukrainische. Es ist seit drei Tagen auf dem Markt. Wir werden sehen, wie die Resonanz sein wird.

GISBERTZ: Schreiben Sie schon weiter, und wenn ja, woran?

WODIN: Ich habe angefangen, an Erzählungen zu arbeiten.

GISBERTZ: Dazu wünschen wir Ihnen viel Kraft, und ich bedanke mich ganz herzlich für das Gespräch.

\section{Literatur}

Hoge-Benteler, Boris (2012): Schreiben über Rußland. Die Konstruktion von Raum, Geschichte und kultureller Identität in deutschen Erzähltexten seit 1989. Heidelberg.

SUREN, KATJA (2011): Ein Engel verkleidete sich als Engel und blieb unerkannt. Rhetoriken des Kindlichen bei Natascha Wodin, Herta Müller und Aglaja Veteranyi. Frankfurt a.M.

Wodin, NATASCHa (1983): Die gläserne Stadt. Eine Erzählung. Reinbek bei Hamburg. Wodin, NATASCHA (1989): Einmal lebt ich. Roman. München.

Wodin, NATASCHA (1993): Erfindung einer Liebe. Roman. Leipzig.

Wodin, Natascha (1997): Die Ehe. Roman. Leipzig.

Wodin, Natascha (2001): Das Singen der Fische. Erzählungen. Leipzig. 
Wodin, Natascha (2009): Nachtgeschwister. Roman. München.

Wodin, NATASCHA (2017): Sie kam aus Mariupol. Reinbek bei Hamburg.

Wodin, NATASCHA (2018): Irgendwo in diesem Dunkel. Reinbek bei Hamburg.

\section{Internetquellen}

https://europamorgenland.de.

https://de.wikipedia.org/wiki/Natascha_Wodin.

https://www.rowohlt.de/autor/natascha-wodin.html.

https://www.zwangsarbeit-archiv.de.

JuRY DES LeIPZIGER BuCHPREISES (2017): Begründung der Preisträgerin 2017-Natascha Wodin. http://www.preis-der-leipziger-buchmesse.de/de/Archiv/2017/ (29.01.2020).

o.A.: Natascha Wodins ,Irgendwo in diesem Dunkel“ ist Ö1 Buch des Monats Dezember. Pressemitteilung des Rowohlt-Verlags vom 26.11.2018. https:/www.rowohlt.de/news/natascha -wodin-irgendwo-in-diesem-dunkel-ö1-buch-des-monats-dezember.html (29.01.2020).

SCHMidTKUNZ, ReNATA (2017): Da capo: Im Gespräch mit Natscha Wodin. https://oe1.orf.at/programm/20170224/460768 (20.01.2020). 